\title{
IoT enabled Electric Vehicle's Battery Monitoring System
}

\author{
Mohammad Asaad $^{1}$, Furkan Ahmad ${ }^{1}$, Mohammad Saad Alam ${ }^{1}$, Yasser Rafat ${ }^{2}$ \\ ${ }^{1}$ Department of Electrical Engineering, Aligarh Muslim University, India \\ \{mohammad.asaad, furkanahmad, saad.alam\}@zhcet.ac.in \\ ${ }^{2}$ Department of Mechanical Engineering, Aligarh Muslim University, India \\ yasser.rafatezhcet.ac.in
}

\begin{abstract}
The Internet of Things (IoT) technology has immense potential for application in improvement and development of Smart Grid. The rising number of distributed generation, aging of present grid infrastructure and appeal for the transformation of networks have sparked the interest in smart grid. The need for energy storage system primarily the electrical energy storage systems is growing as the prospects for their usage is becoming more compelling. Dynamic electrical energy storage system viz., Electric Vehicles (EVs) are relatively standard due to their excellent electrical properties and flexibility but the possibility of damage to their batteries is there in case of overcharging or deep discharging and their mass penetration profoundly impacts the grid. To circumvent the possibility of damage, EVs' batteries need a precise state of charge estimation to increase their lifespan and to protect the equipment they power. Based on ease of implementation and less overall complexity, this paper proposes a real-time Battery Monitoring System (BMS) using coulomb counting method for SoC estimation and messaging based MQTT as the communication protocol. The proposed BMS is implemented on hardware platform using appropriate sensing technology, central processor, interfacing devices and the Node-RED environment. An optimization model aimed at maximizing the trade revenue for EVs' aggregator is presented aimed at enabling the smart charging.
\end{abstract}

Keywords: Internet of Things, Battery Monitoring System, MQTT, SOC estimation.

\section{Introduction}

Internet of Things (IoT) signifies the network based interconnection of daily usage entities. It is termed as a self-organizing wireless linkage of devices aimed at the interconnection of everyday objects. It links with the wireless network through the interface by the electronic identifiers, sensors, two-dimensional codes on things. The IoT technology helps achieve the communication between man and machine or machine to machine. Three key features of IoT are: considerable, intelligent and internet connective [1]. There are four features in IoT: gathering of data, bilateral communication, handling and response control.

The International Telecommunication Union (ITU) laid onward officially the notion of Internet of Things in year 2005 [2]. The report illustrated a draft for the IoT period: when a driver maneuvers erroneously, the vehicle will spontaneously alarm; 
suitcase will prompt the owner about neglecting something; communication from garments to washer about the color and temperature desired and so on.

Since the inception of the smart grid concept being an integral part of IoT was put forward, it has been vastly appreciated by utilities. IoT integrated Smart Grid results in improvement of energy productivity, reduced ecological influence, enhanced security, reduced vulnerability to external interference and increased consistency of electric supply [3].

The integration of Smart Grid tools in utility networks will influence marvelous transformation in grid management and electric power use in upcoming years. The methodical change in load regulation, along with boosted dispersion of nonconventional energy sources, suggest a fresh array of challenges in equalizing expenditure and production. Increased deployment of energy storage devices in the distribution grid will help expedite this process and improve system performance [4]. Bulk energy storage has been used for decades in the utility grid and now the integration of renewables is creating a need for more distributed storage. With increasing adoption of non-conventional energy sources and rise in popularity of plugin hybrid electric vehicles (PHEVs) and all electric vehicles (EVs), the need is for a far more vigorous electric infrastructure. Figure 1 shows the key areas where energy storage systems can be applied.

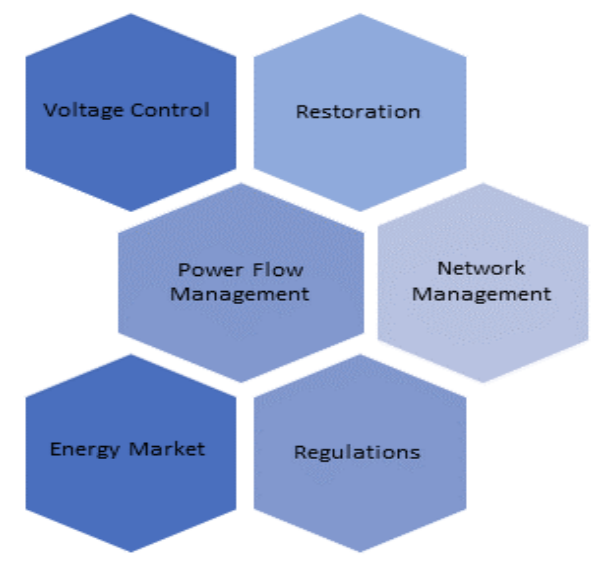

Fig. 1: Areas of application of energy storage in smart grid

Innovations in battery technology have been the key motivation for distributed storage systems. With the increasing penetration of electric mobility, the battery prices are declining which will be of assistance in grid applications. To prolong the life of battery-based energy storage system and ensure their reliability, a proper battery monitoring system needs to be integrated along with.

This paper proposes a lightweight communication protocol based EV's battery monitoring system aimed at prolonging the battery pack's useful life. The paper is organised as follows. Section II sheds light on EVs impact on grid and how bilateral flow of power can improve grid functionality. Section III tells key features of a typical battery monitoring system. Section IV gives background information on battery capacity estimation technique used. Section V provides the framework of proposed hardware setup. Results are shown in Section VI. To reduce the impact of 
uncontrolled charging of EVs on grid, an optimization model intended to maximize the EV's aggregator revenue is formulated in Section VII.

\section{Electric Vehicles and Smart Grid}

As per reports of National Renewable Energy Laboratory (NREL), mass production of EVs has limited adverse effect on the electricity generation need. For example in USA, for EVs constituting the $50 \%$ of the total vehicles in use by year 2050 will entail only $8 \%$ increment in electricity generation and an increment of $4 \%$ in generation capacity meanwhile also considerably reducing the emissions from conventional vehicles and lowering the fuel usage in transportation sector [5]. Other effect of mass adoption of EVs on power grid are-

- Increase in the working temperature of transformers due to the extra load of charging EVs. This reduces the operating life, thereby incurring additional expenditure.

- The energy storage system ought to store electricity from the minimum carbon producing sources, e.g., nuclear energy and renewable energy. However, coinciding the demand and supply load curves is a big challenge.

- Shortage in electric power supply, if the accumulated EV charging profile constitutes the peak demand period.

However, charging of EVs at off peak hours augments the load curve for electric utilities. So, the usage of large no of EVs must be accurately optimized for various charging setups and technologies. A sample optimization model for smart charging is presented in section VII in this paper.

Demand Response (DR) is additional advantage to the grid by disrupting the EVs charging demand at peak hours. In parked condition, EVs generate or store electricity which can be fed back to grid using appropriate connections- this is known as vehicleto-grid power or $\mathrm{V} 2 \mathrm{G}$ power. The batteries of EVs plugged into charging infrastructure can act as distributed energy storage systems for the electrical grid. The electrical energy delivered backed to the grid must be priced such that the additional cost incurred must be recovered back as the battery's lifecycle is reduced due to frequent charging-discharging. The distributed storage provides advantages such as making the grid more steady, secure and resilient by regulating frequency and the spinning reserve as backup power in the distribution system. Large scale integration of intermittent sources of energy e.g., wind and solar sources into the grid is facilitated by V2G system. For the world-wide shift to the emerging green and sustainable energy economy, V2G is an important enabler.

\section{Battery Monitoring System}

Battery Monitoring System (BMS) is a smart system whose function is to monitor the vigor of a battery pack. BMS computes the battery's capacity, depreciation of battery while the charging/discharging and correct productivity of the battery and provides this information in real time to users. This mitigates the sense of incorrect safety of periodic battery assessment as it is vigilant to emerging issues before hand 
the occurrence of a possible malfunction. As every cell is observed separately, so any damage can be checked and appropriate warnings against the values pre-set by consumers and protective measures can be employed, safeguarding the other cells against cumulative damage thereby extending battery life. BMS logs history data of all measured parameters for further analysis and future reference.

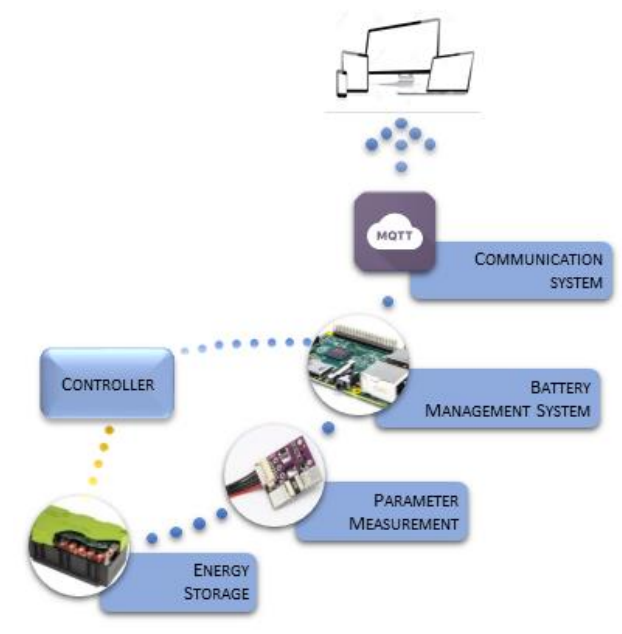

Fig. 2: A BMS Framework

Figure 2 shows a typical BMS framework incorporating the measurement of key battery parameters e.g., current, voltage, temperature etc., and performing necessary calculations/estimations to extract useful information about energy storage system i.e., State of Health $(\mathrm{SoH})$, State of Charge (SoC), operating temperature range. Based on these calculated parameters, controlling actions are taken to maintain the battery's lifecycle and safety against potential hazards. Therefore, the prime objective of monitoring is to gauge various variables, log events, generate warnings, record usage profile and represent this information locally and remotely to the user.

BMS is unable to sense movable connections present in the battery, leakage of cell material, corrosion of connections leading to the development of high resistance and subsequently fire danger. It is also unable to visually monitor developing swelling, potential leakage, cracks in the outer geometry of battery pack etc.

\section{SoC Estimation}

Proper battery use demands the knowledge of its State of Charge (SoC). The development of suitable control strategy necessitates the accurate estimation of battery's remaining capacity i.e., SoC [6]. Being a vital parameter depicting battery performance, precise estimation prolongs the battery lifecycle, avoids deep discharges and helps designing practical control methods to keep battery operating in the optimum region. However, a battery being a chemical energy storage source, and this chemical energy isn't directly accessible which makes the estimation of the $\mathrm{SoC}$ of a battery challenging [7]. By estimating the present capacity of the battery, it can be 
safely charged/discharged at levels appropriate for battery lifecycle enhancement. The energy capacity of a battery depends upon its charging current, discharging current, oldness, operating temperature, cut-off voltage, and usage profile.

Numerous techniques have been suggested for the batteries SoC estimation [3-4]. These techniques can be classified into three types: electrochemical-based, electrical based and adaptive ones. The electrochemical techniques although highly accurate are considered difficult to implement in software or hardware as they require access to the chemical composition of battery. Adaptive techniques [7] involve a battery equivalent model and a solution algorithm e.g., neural network [10], Kalman filter [11] and fuzzy logic algorithm. The efficiency of the equivalent model determines the accurateness of these techniques. But, electrical techniques demand only measurable parameters e.g., terminal voltage, charge/discharge current and internal resistance. Due to ease of implementation and low complexity, coulomb counting technique which is based on the integration of current over time is one of the most commonly used electrical technique for SoC estimation [10,11].

In general, battery's SoC is termed as the proportion of its current capacity $(Q(t))$ to the nominal capacity $(Q n)$. The battery manufacturer specifies the nominal capacity which shows the utmost quantity of charge that can be stored in the battery. The SoC can be defined as follows:

$$
\operatorname{SoC}(t)=\frac{Q(t)}{Q_{n}}
$$

\subsection{Coulomb Counting Method}

With assumption that initial SoC (at time $t_{o}$ ) is in knowledge, SoC at any instant is usually estimated by integration of the battery current over time, as shown in equation (2)

$$
\operatorname{SoC}(t)=\operatorname{SoC}\left(t_{o}\right)+\frac{\int_{t}^{t+\Delta} I_{b a t .} d \Delta}{Q_{n}} \times 100 \%
$$

SoC : State of Charge, $I_{b a t}$ : value of battery current, $Q_{n}$ : nominal capacity

The accurateness of Coulomb counting technique depends upon various parameters viz., operating temperature, battery usage history, discharge current, and cycle life [14].

The coulomb counting technique consists of using the equation (2) by enumerating the charge supplied by the battery by sensing its input and output current [10]. Though, few inefficiencies are there in this technique- the initial $\mathrm{SoC}$ value is not correctly known, presence of self-discharge phenomena can change the real SoC value after a prolong storage time and battery degradation due to aging should be taken into consideration.

\section{Hardware Setup}

Rechargeable battery frameworks are to be an essential part of energy storage system in smart grids. Monitoring of battery performance is one of the key issue in 
control and management of a battery management system. The measurement part of BMS records cell voltages, battery current, and converts them into the digital measure. The measurements are done in every specimen period and communicated with a processor through an Analog-Digital (A-D) interface. Using these deliberate battery parameters, the processor's function is to assess the SoC. Although SoC is considered as the "fuel gauge" of the energy storage system, but it actually displays the remaining vitality in the battery.

The coulomb counting technique for SoC estimation was realized on the hardware platform. The battery monitoring system include the measurement of battery parameters viz., voltage, current flow and temperature by appropriate sensing technology and interfaced through an ADC (Analog to Digital Converter) device to microcontroller Broadcom BCM2837 SoC based Raspberry Pi 3 Model B. The SoC estimation technique has been implemented by measuring voltage, current and temperature of two parallel connected 3S Li-Po (Lithium Phosphorus) batteries with total nominal capacity of $4400 \mathrm{mAH}$. The direction of flow of current determines whether the battery is in charging or discharging condition. When the current ceases to flow, it is assumed that battery is in open circuit condition i.e., idle provided recent SoC monitored is more than $10 \%$. If so, the last SoC reported is taken as initial SoC for next cycle. If SoC falls down below 5\%, it is assumed that batteries are completely discharged and SoC is set to $0 \%$ for next cycle. Similarly, algorithm is applied at full charge condition. The estimated SoC along with experimentally obtained parameters by the BMS were shown both on PC and MQTT Broker-DIoTY App for Smartphone. The work can be further extended by integration of database management system to predict the time to charge/discharge the battery.

\subsection{Node-Red}

The estimation algorithm was implemented in the Node-Red environment. NodeRED is a graphical means for connecting various hardware appliances, Application Programming Interfaces (APIs) and real-time facilities together- to equip the Internet of Things. Using a browser based flow editor, Node-RED offers an extensive range of nodes to simply connect the flows which can be executed to the runtime with minimal effort. The light-weight runtime is built on Node.js, taking maximum benefit of its event-driven, non-blocking model [15].

\subsection{MQTT}

MQTT (Message Queuing Telemetry Transport) is a messaging based communication protocol that affords the lightweight network with an easy means to deliver data. The protocol is used for machine-to-machine (M2M) communication and plays an imperative part in the IoT. It uses a publish/subscribe communication model.

MQTT is a useful selection for wireless systems which undergo fluctuating levels of latency because of bandwidth restrictions or fickle connections.

In publish/subscribe model, communication is straight from client to an endpoint. But the publisher (client sending message) and subscriber (client getting message) have no knowledge about the presence of each another. There exist a third element, 
known as broker, who is familiar with both the existing parties i.e., publisher and subscriber. The broker categorizes every received message and delivers them suitably. As MQTT delinks the publisher and subscriber, only the information about hostname/IP and port of the broker is sufficient in order to communicate with messages. Delivery success is effortlessly conveyed upon the successful communication of the message [16].

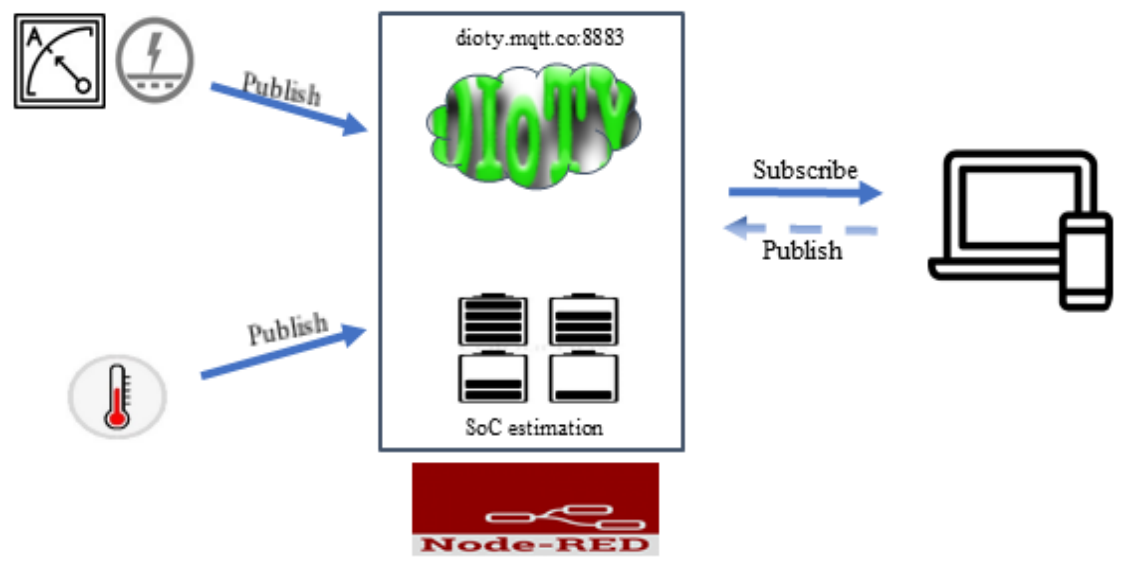

Fig. 3: BMS Hardware Block Diagram

Figure 3 shows the BMS hardware prototype at the block level. The battery packs electrical parameters are measured using hall effect current/voltage sensors and after amplification, are communicated with the processor Raspberry Pi 3 B interfaced through an 8-bit ADC. Likewise, the external temperature is measured using sensor DHT 11 and conversed with the processor. The coulomb counting algorithm is implemented in the Node-RED environment and estimated SoC is communicated via MQTT protocol. Users can view the value of SoC and operating temperature in smartphone or PC by subscribing to corresponding topics via the broker DIoTY.

The lifecycle of the battery pack can be augmented overall by the feasible formulation of battery charging, discharging, and sleep practices e.g., in the occurrence of SoC topping 10\%, the discharge should be allowed and in the occurrence that it trips down below $10 \%$, the discharge need to be stopped. When the SoC touches $95 \%$, then the battery charging must be stopped. Cyclic full charge/discharge enhances the longevity of the battery pack. The operation should be ensured below the specified temperature to avert the risk of explosion of the battery.

\section{Results}

The working of SoC estimation technique and real time of communication of battery parameters were tested during the charging mode of the LiPo batteries. Figure 4 represents the effective capacity estimated by the coulomb counting method. These values are communicated with user using MQTT protocol. 


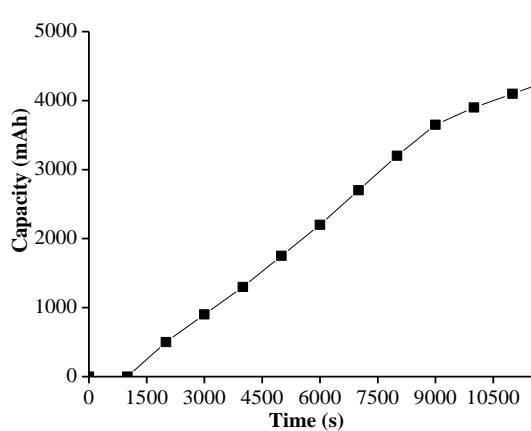

Fig. 4: Estimated capacity of battery packs

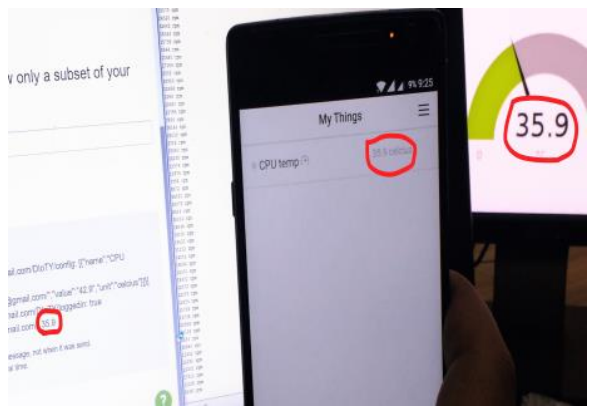

Fig. 5: Battery pack temperature across different platforms

The battery external temperature measured is communicated in real time. Figure 5 shows the temperature displayed on the PC and smartphone App labeled as CPU temp using DIoTY broker.

\section{Smart Charging}

Optimal scheduling of EVs charging is a necessity to counter the increasing burden on grid. If not done in advance, it will lead to imbalance of voltage as well as frequency, which ultimately may lead to grid failure and blackout. Continuous monitoring of power system is necessary while charging large number of EVs, in order to achieve grid balancing. A typical demand profile for one day in terms of $15 \mathrm{~min}$ bidding time blocks, obtained from Indian Power Exchange (IEX) is shown in Fig. 6 which is a replica of the energy demand in Indian scenario, where the minimum load is observed during the night hours and also during the mid of the day.

Low peak hours during night, wherein the load is at its minimum, are most suitable for home based charging, using either Level 1 or Level 2. This period of 6 to 8 hours is also very good for charging batteries at Battery Swapping Stations (BSS). Public charging stations (CSs), where most of the charging is expected to occur during day time, can shift their charging load to low peak hours to enhance the functioning of grid. This can be done by deploying attractive charging schemes for the consumers during low peak hours or by storing energy during this period as a backup for peak load hours. This type of scheduling can prove to be beneficial for both the utility and consumers. A flatter load profile with less system peak differences can be obtained this way, which is desirable. Also, from the consumer's view point, this is advantageous as evident from Fig. 6. Cost of purchasing electricity is lower during low peak hours as compared to that during peak hours.

Such a demand side management can help in smooth running of power grid with fewer disturbances. Further enhancement of grid operation can be achieved by injection of power back to the grid and also by participating in reserve market to maintain the frequency up and down regulation. This can prove to be beneficial for the EV aggregator as well as to consumers, in terms of economy.

The aggregator of EV charging station, as well as BSS, can gain maximum profit by optimizing the cost function of trading revenue mentioned in [17] which includes 
day ahead (DA) market, reserve market (i.e. regulation up and down) and real time market (unscheduled interchange).

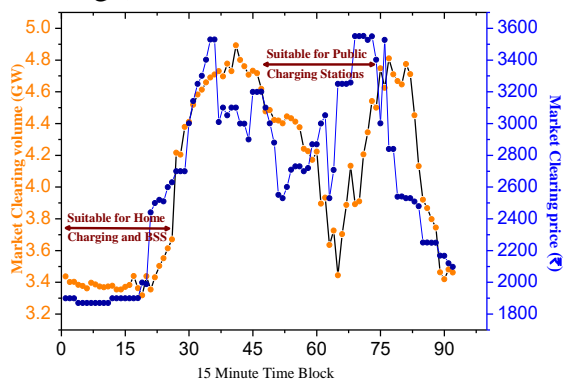

Fig. 6: Demand profile for one day

In order to participate in DA market, proper load forecasting is essential. Numerous techniques have been developed for load forecasting like Simplistic Benchmark methods, Seasonal ARMA modeling, Periodic AR models etc. [18].

The uncertainties involved in the EV fleet characteristics, DA electricity market operations as well as in generation, transmission, and load may be represented by Monte Carlo simulation. Risk involved with the financial as well as economical aspects of the EV aggregator in uncertainty environments can be managed by conditional value added risk analysis (CVaR) as given in [19].

\section{Conclusion}

Internet of Things (IoT) refers to the networked interconnection of everyday objects. IoT has a major role in the rapid development of smart grid. The implementation of Smart Grid devices in the utility grid will influence vast modification in grid management and usage of electric power in upcoming years. The integration of distributed generation necessitates the deployment of energy storage system. Due to better electrical characteristics, the dynamic energy storage system i.e., Electric Vehicles (EVs) is a good prospect although the probability of damage to battery pack in case of overcharging or deep discharging situations is there and uncontrolled charging can severely impact the grid functioning. To mitigate the danger of damage, an accurate real-time capacity determination of a battery pack is desired to increase their lifespan and to protect the equipment they power. A less complex and easy to implement algorithm i.e., coulomb counting technique is implemented in this paper and the estimated SoC along with measured parameters are made available in real time to the user on a remote basis in form of messaging communication. Further an optimization model for maximizing the trade revenue for aggregator of EVs is presented aimed at facilitating smart charging to reduce the impact of increased penetration of EVs on grid.

\section{Acknowledgement}

This research is supported by the Centre of Advanced Research in Electrified Transportation (CARET), Aligarh Muslim University, India sponsored by the grant from the Ministry of Heavy Industries, Govt. of India under FAME Mission. 


\section{References}

[1] K. Guo Dengfeng, Xu Shan, "The Internet of Things hold up Smart Grid networking technology," North China Electr., vol. 2, pp. 59-63, 2010.

[2] ITU, "The Internet of Things," Itu Internet Rep. 2005, p. 212, 2005.

[3] X. Fang, S. Misra, G. Xue, and D. Yang, "Smart Grid - The New and Improved Power Grid: A Survey,” IEEE Commun. Surv. Tutorials, vol. 14, no. 4, pp. 944-980, 2012.

[4] B. P. Roberts and C. Sandberg, "The role of energy storage in development of smart grids," in Proceedings of the IEEE, 2011, vol. 99, no. 6, pp. 1139-1144.

[5] R. Liu, L. Dow, and E. Liu, "A survey of PEV impacts on electric utilities," in IEEE PES Innovative Smart Grid Technologies Conference Europe, ISGT Europe, 2011.

[6] Z. Rao, S. Wang, and G. Zhang, "Simulation and experiment of thermal energy management with phase change material for ageing LiFePO4 power battery," Energy Convers. Manag., vol. 52, no. 12, pp. 3408-3414, 2011.

[7] N. Watrin, B. Blunier, and A. Miraoui, "Review of adaptive systems for lithium batteries state-of-charge and state-of-health estimation," in 2012 IEEE Transportation Electrification Conference and Expo, ITEC 2012, 2012.

[8] S. Piller, M. Perrin, and A. Jossen, "Methods for state-of-charge determination and their applications," in Journal of Power Sources, 2001, vol. 96, no. 1, pp. 113-120.

[9] L. Lu, et.al., "A review on the key issues for lithium-ion battery management in electric vehicles," Journal of Power Sources, vol. 226. pp. 272-288, 2013.

[10] M. Charkhgard and M. Farrokhi, "State-of-charge estimation for lithium-ion batteries using neural networks and EKF," IEEE Trans. Ind. Electron., vol. 57, no. 12, pp. 4178-4187, 2010.

[11] G. Y. Y. Ding-xuan, "SOC estimation of Lithium-ion battery based on Kalman filter algorithm," in 2nd International Conference on Computer Science and Electronics Engineering (ICCSEE), 2013.

[12] C. M. and Y. H. K.S. Ng, Y.F Huang, "An enhanced coulomb counting method for estimating state-of-charge and state-of-health of lead-acid batteries," in 31st International Telecommunications Energy Conference (INTELEC 2009).

[13] Y. M. Jeong, Y. K. Cho, J. H. Ahn, S. H. Ryu, and B. K. Lee, "Enhanced coulomb counting method with adaptive SOC reset time for estimating OCV," in 2014 IEEE Energy Conversion Congress and Exposition, ECCE 2014, 2014, pp. 4313-4318.

[14] K. S. Ng, et.al., "Enhanced coulomb counting method for estimating state-of-charge and state-of-health of lithium-ion batteries," Appl. Energy, vol. 86, no. 9, pp. 15061511,2009

[15] Node-Red.: http://nodered.org/.

[16] MQTT Publish \& Subscribe.: http://www.hivemq.com/blog/mqtt-essentials-part2publish-subscribe.

[17] M. R. Sarker, Y. Dvorkin, and M. A. Ortega-Vazquez, "Optimal participation of an electric vehicle aggregator in day-ahead energy and reserve markets," IEEE Trans. Power Syst., vol. 31, no. 5, pp. 3506-3515, 2016.

[18] J. W. Taylor and P. E. McSharry, "Short-term load forecasting methods: An evaluation based on European data," IEEE Trans. Power Syst., vol. 22, no. 4, pp. 2213-2219, 2007.

[19] H. Wu and M. Shahidehpour, "A Game Theoretic Approach to Risk-Based Optimal Bidding Strategies for Electric Vehicle Aggregators in Electricity Markets With Variable Wind Energy Resources," vol. 7, no. 1, pp. 374-385, 2016. 